\title{
Interaction of Fluorescent Gold Nanoclusters with Transition Metal Dichalcogenides Nanosheets: A Spectroscopic Study
}

\author{
Arun Singh Patel \\ School of Computational \& Integrative Sciences, Jawaharlal Nehru University, \\ New Delhi-110067, India and \\ Department of Physics, Hindu College, University of Delhi, \\ New Delhi-11000 'a) \\ Anirban Chakraborti \\ School of Computational \& Integrative Sciences, Jawaharlal Nehru University, \\ New Delhi-110067, India and \\ Centro Internacional de Ciencias, Cuernavaca-62210, Méxicd ${ }^{\mathrm{b})}$ \\ Praveen Mishra \\ School of Computational $\&$ Integrative Sciences, Jawaharlal Nehru University, \\ New Delhi-110067, India
}

In this paper, the interaction of few tens of atoms containing gold nanoclusters with two dimensional nanosheets of transition metal dichalcogenides nanosheets has been explored. The gold nanoclusters have been synthesized using chemical reduction method in presence of protein molecules as stabilizing agent. The transition metal dichalcogenides nanosheets of molybdenum disulfide $\left(\mathrm{MoS}_{2}\right)$ has been chemically exfoliated. Different microscopic and optical spectroscopic tools have been used for characterizing the physical properties of the gold nanoclusters and the two dimensional nanosheets of $\mathrm{MoS}_{2}$. The gold nanoclusters exhibit fluorescence emission at $690 \mathrm{~nm}$. However, the interaction with transition metal dichalcogenides diminishes drastically the fluorescence intensity of the nanoclusters. The spectroscopic methods used for understanding the interaction in the system reveals the absence of energy transfer and dynamic nature of the fluorescence quenching.

\section{INTRODUCTION}

In recent years, the metal nanoclusters (NCs) consisting of few tens of metal atoms have been studied extensively due to their intriguing physical properties and excellent chemical stability! 15 The size of these nanoclusters lies in between individual atoms and nanoparticles. These metal nanoclusters provide a bridge between atomic and nanoparticles behavior of noble metals ${ }^{6 / 7}$ At this length scale, the size of nanoclusters is comparable to the Fermi wavelength of the free electrons of the metal, which results in quantum confinement of the free electrons in the nanoclusters. The quantum confinement leads to widening of the energy gap between the discrete electronic energy levels. The discrete energy levels enable these nanoclusters to act as highly optically active materials in the visible range, which is generally absent in the case of nanoparticles and the bulk metal. Similar to the fluorescent dye molecules, gold nanoclusters also show strong fluorescence at low temperature as compared to high temperature. The relationship among the energy of emitted photons $\left(E_{g}\right)$ from metal nanoclusters and number of atoms $(N)$ and the Fermi energy $\left(E_{F}\right)$ of metal can be represented in the following form 8

$$
E_{g}=\frac{E_{F}}{N^{1 / 3}} .
$$

\footnotetext{
a) Electronic mail: arunspatel.jnu@gmail.com
}

b) Electronic mail: anirban@jnu.ac.in 
Gold nanoclusters are less toxic as compared to other metal nanoclusters. Hence, gold nanoclusters can be a good choice as fluorescent materials in comparison with available fluorescent dye molecules. The dye molecules that are used as fluorescent tags in spectroscopic analysis have toxic nature; so, they have limitations in direct use in biological studies. In recent years, there has been great interest among the researchers to understand the interaction of fluorescent materials with different kinds of nanomaterials, starting from dye molecules with quantum dots to quantum dots with $2 \mathrm{D}$ nanomaterials ${ }^{9} 14$ These interactions have been explored for different areas of application like sensing, solar cell, imaging, etc. Since discovery of graphene, people started searching for other forms of 2D nanosheets and in recent times different 2D nanomaterials, which have varying band gap from UV to IR region, are being extensively studied. Among these 2D nanosheets, the nanosheets of transition metal dichalcogenides (TMDCs), which have band gap in the visible wavelength range found great interest 15 These TMDCs possess indirect band gap when they are in bulk form and show direct band gap while in single layer form.20 22 Among these TMDCs nanosheets, molybdenum disulfide $\mathrm{MoS}_{2}$ and tungsten disulfide $\mathrm{WS}_{2}$ have been studied extensively. The nanosheets of $\mathrm{MoS}_{2}$ have number of layers dependent bad gap and hence the band gap can be tuned easily. This attracted tremendous attention due to its intriguing chemical, physical and mechanical properties. Good biocompatibility, flexible surface and excellent fluorescence quenching capability have made $\mathrm{MoS}_{2}$ compatible for numerous applications ${ }^{23} \sqrt[25]{25}$ Understanding the interaction between $\mathrm{MoS}_{2}$ and gold nanoclusters is the key factor for the development of biosensors, drug delivery and therapeutic systems. In this paper, the interaction of gold nanoclusters with $\mathrm{MoS}_{2}$ nanosheets has been investigated. This work is important for exploring the possibilities of energy transfer in nanomaterials where biomolecules are present at the interface of different nanomaterials, which can be further explored for sensing applications.

\section{METHODS AND RESULTS}

Synthesis of gold nanoclusters: For synthesis of gold nanoclusters, chloroauric acid $\left(\mathrm{HAuCl}_{4} \cdot 3 \mathrm{H}_{2} \mathrm{O}\right.$, Sigma Aldrich) was used as precursor of gold nanoclusters. Gold nanoclusters were synthesized using chemical reduction method. In typical synthesis procedure, 50 $\mathrm{mg}$ bovine serum albumin (BSA) was dissolved in $1 \mathrm{~mL}$ of distilled water. In the BSA solution, $1 \mathrm{~mL}$ of $10^{-2} \mathrm{M}$ gold salt aqueous solution was added, and the mixture was stirred for $5 \mathrm{~min}$. Further, $0.1 \mathrm{~mL}$ aqueous solution of $1 \mathrm{M}$ sodium hydroxide was injected into the solution, and the mixture was allowed to be stirred for $10 \mathrm{~min}$. As-prepared solution was incubated at $37{ }^{0} \mathrm{C}$ for $24 \mathrm{~h}$. The yellow colored solution turned to a reddish-brown after formation of gold nanoclusters. The aqueous dispersion of gold nanoclusters was kept at 4 ${ }^{0} \mathrm{C}$ for further study.

Chemical exfoliation of $\mathrm{MoS}_{2}$ nanosheets: The $\mathrm{MoS}_{2}$ nanosheets were obtained by chemical exfoliation of the bulk $\mathrm{MoS}_{2}$ powder using ultrasonication technique. For this purpose, a stock solution of BSA was prepared $(1 \mathrm{mg} / \mathrm{mL})$. The solution was used as solvent for dispersion of bulk $\mathrm{MoS}_{2}$ powder. $50 \mathrm{mg}$ of $\mathrm{MoS}_{2}$ powder was dispersed in $10 \mathrm{~mL}$ of the aqueous solution of BSA. The dispersion of $\mathrm{MoS}_{2}$ was ultrasonicated for $20 \mathrm{~h}$. After ultrasonication, the precipitate was allowed to settle down and the supernatant was collected, and a stock solution of $\mathrm{MoS}_{2}$ was made.

Characterization techniques: The gold nanoclusters and $\mathrm{MoS}_{2}$ nanosheets were characterized by different spectroscopic and microscopic techniques. The absorption spectra of these nanomaterials were recorded using UV-visible spectrophotometer (IMPLEN, Inkarp). The Raman spectrometer from WITEC was used to investigate the Raman active vibrational modes of $\mathrm{MoS}_{2}$. For this purpose, Raman spectrometer, with $532 \mathrm{~nm}$ laser excitation source was used. The shape and size of the nanosheets were analyzed by transmission electron microscope (JEOL-2010) operated at $200 \mathrm{keV}$. The fluorescence spectra of gold nanoclusters in absence and presence of $\mathrm{MoS}_{2}$ nanosheets were recorded using fluorimeter. The fluorescence lifetime decay was monitored using time correlated single photon counting 


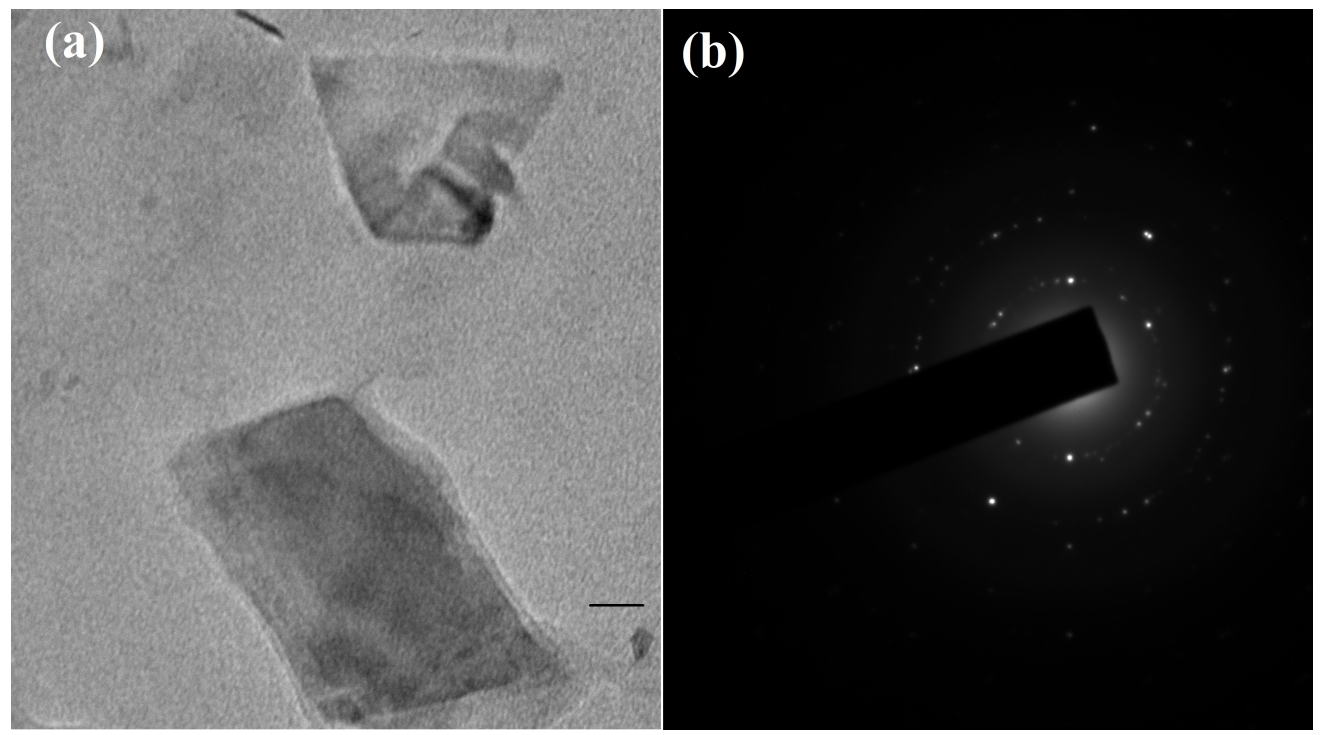

FIG. 1. TEM images of (a) $\mathrm{MoS}_{2}$ nanosheets, and (b) the selective area electron diffraction pattern of the nanosheets, scale bar equals to $20 \mathrm{~nm}$.

set-up (FL920, Edinburgh Instruments, UK). The excitation source was $370 \mathrm{~nm}$ pulse laser with operating frequency of $20 \mathrm{MHz}$.

The shape and morphology of $\mathrm{MoS}_{2}$ nanosheets were monitored by TEM analysis. The TEM image of $\mathrm{MoS}_{2}$ nanosheets is shown in Figure 1, where sheet like structure of $\mathrm{MoS}_{2}$ is observed. A color contrast in the TEM image is observed in Figure 1(a), which arises due to few layers of the nanosheets. Figure 1(b) is the selective electron diffraction (SAED) pattern, where hexagonal spots are observed due to the hexagonal lattice structure of $\mathrm{MoS}_{2}$.

The Raman spectrum of $\mathrm{MoS}_{2}$ nanosheets is shown in Figure 2. In case of $\mathrm{MoS}_{2}$ nanosheets, there are two distinct Raman peaks, which are observed at 385 and $409 \mathrm{~cm}^{-1}$ and these peaks are assigned as $E_{2 g}^{1}$ and $A_{1 g}$ vibrational modes, respectively. The $E_{2 g}^{1}$ peak originates due to in-plane vibration of the sulfur and molybdenum atoms in the lattice, while the $A_{1 g}$ peak is due to out-of-plane vibration of the sulfur atoms.

The optical absorption of gold nanoclusters and $\mathrm{MoS}_{2}$ nanosheets are recorded using absorption spectrophotometer in the visible range. The absorption spectra of gold nanoclusters, $\mathrm{MoS}_{2}$ and mixture of both are shown in Figure 3. The gold nanoclusters have continuously increasing absorbance towards the lower wavelength of the spectrum. The absorption spectrum of $\mathrm{MoS}_{2}$ exhibits two distinct absorption peaks - one appearing at 613 $\mathrm{nm}$ and the other at $670 \mathrm{~nm}$, which are known as B and A excitonic peaks, respectively.

In order to investigate the effect of the presence of $\mathrm{MoS}_{2}$ on the fluorescence property of the gold nanoclusters, the fluorescence spectra of gold nanoclusters have been recorded, both in absence and presence of different quantities of $\mathrm{MoS}_{2}$ nanosheets. The fluorescence spectra of gold nanoclusters in the absence and presence of $\mathrm{MoS}_{2}$ are shown in Figure 4 . The fluorescence $(\mathrm{Fl})$ emission of gold nanoclusters is observed at $690 \mathrm{~nm}$. In presence of $\mathrm{MoS}_{2}$ nanosheets the Fl intensity of gold nanoclusters diminishes drastically. The Fl quenching may be due to either static or dynamic nature of quenching. ${ }^{26 \mid 27}$ Using equation 2. the Fl quenching efficiency was estimated

$$
E=1-\frac{F_{D A}}{F_{D}}
$$

where $\mathrm{F}_{D A}$ and $\mathrm{F}_{D}$ are fluorescence intensities of gold nanoclusters in presence and absence of $\mathrm{MoS}_{2}$ nanosheets. The Fl quenching efficiency was found to be 41 and $89 \%$ for $\mathrm{MoS}_{2}$ nanosheets with $0.1 \mathrm{~mL}$ and $0.7 \mathrm{~mL}$ of the acceptor quantities, respectively. Further, the nature of the fluorescence quenching was investigated using time resolved fluorescence 


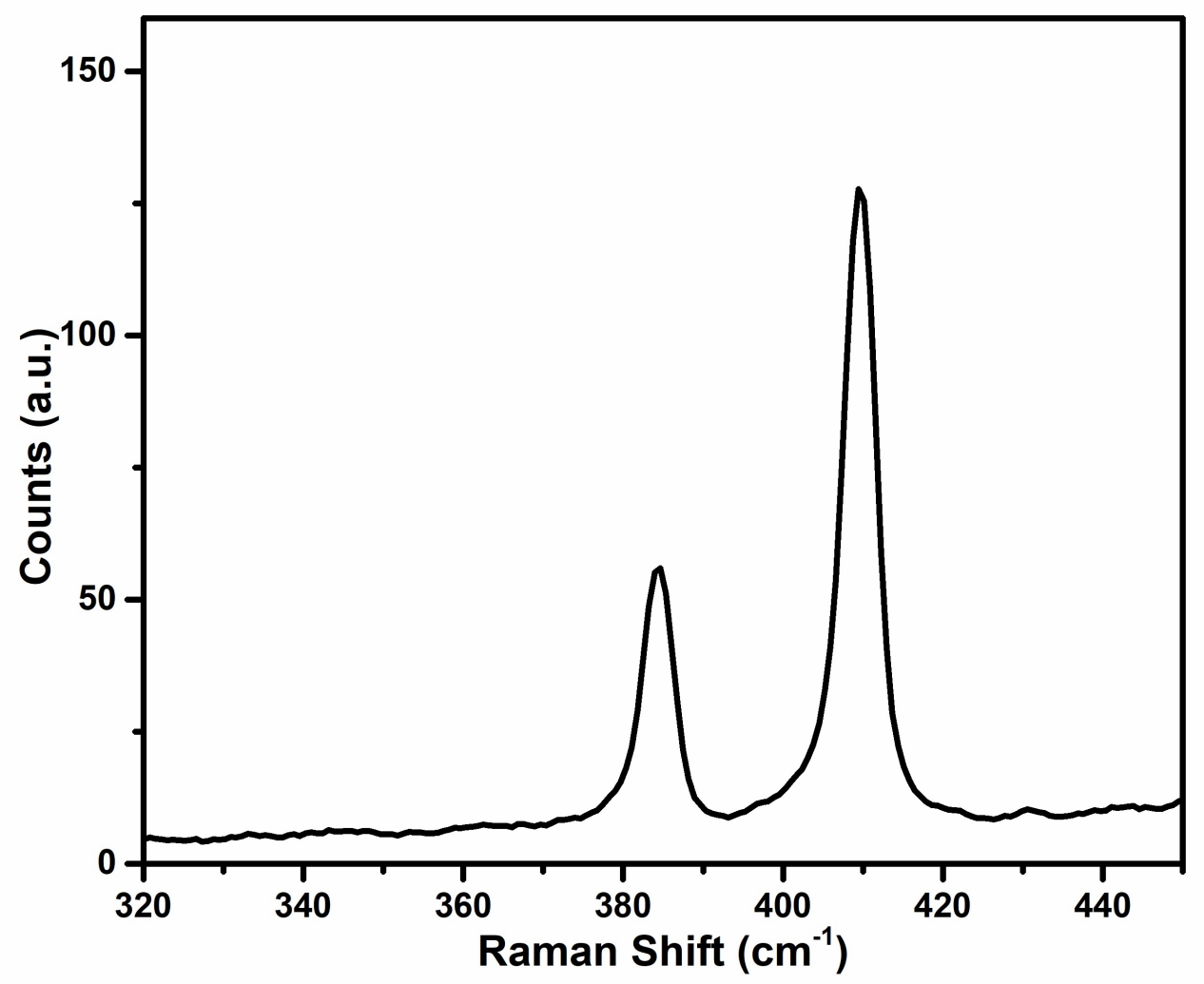

FIG. 2. Raman spectrum of $\mathrm{MoS}_{2}$ nanosheets.

spectroscopic (TRFS) technique. The fluorescence lifetime of the gold nanoclusters was estimated from the fluorescence lifetime decay curve of the gold nanoclusters. The fluorescence lifetime decay curves of gold nanoclusters in absence and presence of the nanosheets, are shown in Figure 5. The fluorescence lifetime decay curves were fitted with exponential decaying function of the following form 28

$$
I(t)=\sum_{j=1}^{m} \alpha_{j} \exp \left(-t / \tau_{j}\right),
$$

where $\alpha_{j}$ is the weighing factor, $\tau_{j}$ is the fluorescence lifetime associated with $j^{\text {th }}$ component. For gold nanoclusters, the Fl decay curves were fitted with triple exponential decaying functions. The three components are associated with three different environments of the emitters. In gold nanoclusters, it has been observed that there are few gold atoms in $\mathrm{Au}(\mathrm{I})$ state, while the emission comes out due to $\mathrm{Au}(0)$ atoms, which are forming core of the gold nanoclusters. The gold nanoclusters are formed with 25 gold atoms, which are in conjugation with BSA molecules. Few gold atoms bind with sulfur atoms of the BSA molecules and thus the inner gold atoms are in $\mathrm{Au}(0)$ state. Thus, one component is arising due to core and the other due to outer interacting nanoclusters. The outer component is responsible for high Fl lifetime value, while the inner one is responsible for small value of the Fl lifetime. The average Fl lifetime of gold nanoclusters is found to be $1.1 \mu \mathrm{s}$. From Figure 5, it is observed that the nature of the decaying curves remains the same in presence of $\mathrm{MoS}_{2}$ nanosheets, even at very high concentrations of the $\mathrm{MoS}_{2}$ nanosheets. Thus, the Fl lifetime remains unaltered in presence of $\mathrm{MoS}_{2}$ nanosheets.

The time resolved fluorescence study shows that there is no change in the fluorescence lifetime of gold nanoclusters in presence of $\mathrm{MoS}_{2}$ nanosheets even at very high concentration 


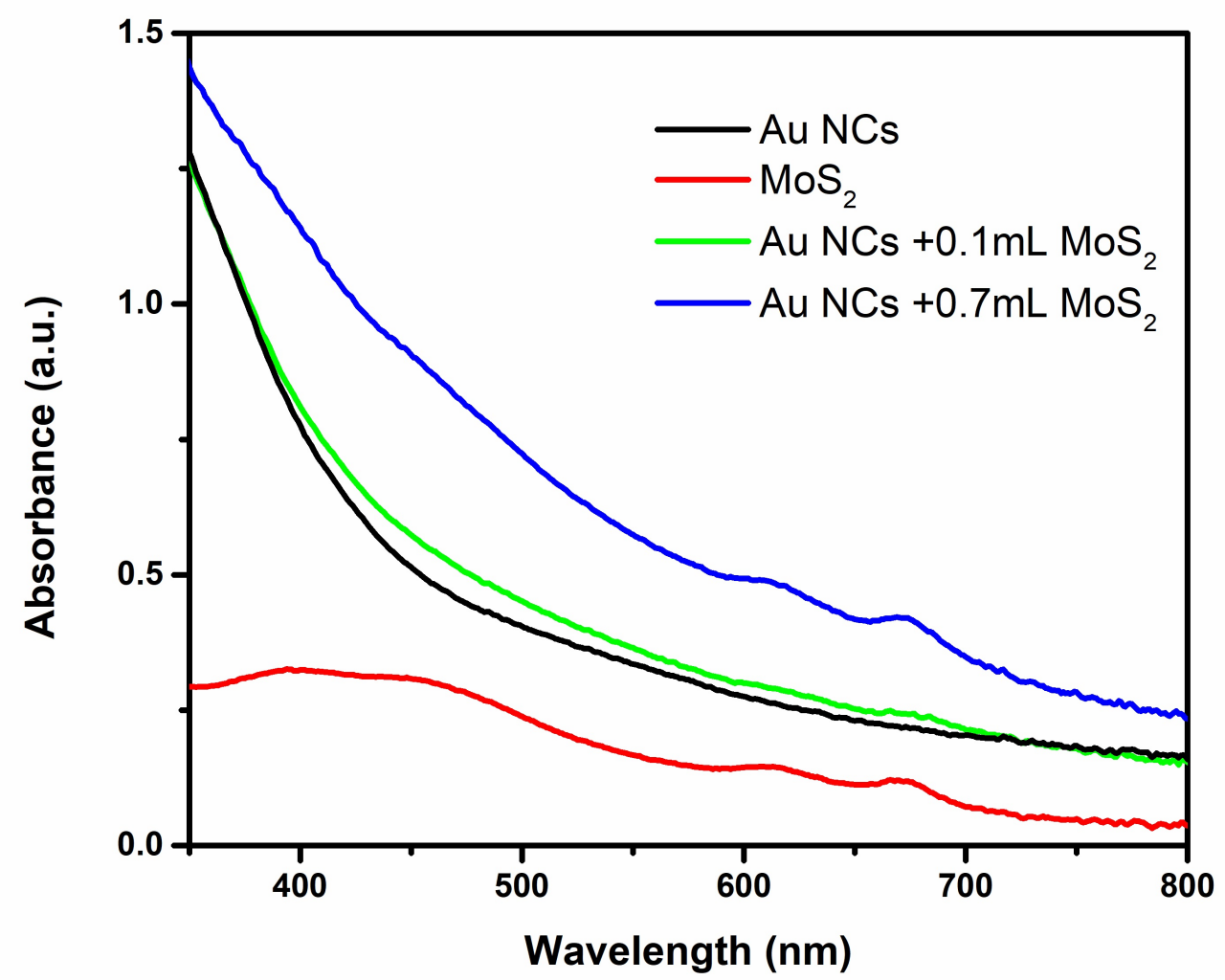

FIG. 3. Absorption spectra of gold nanoclusters, $\mathrm{MoS}_{2}$ nanosheets, and mixed dispersion of gold nanoclusters and $\mathrm{MoS}_{2}$ nanosheets.

of the nanosheets. Previously, it had been reported that the energy transfer from adjacent BSA molecules to the $\mathrm{MoS}_{2}$ nanosheets could possibly occur. ${ }^{29}$ In that case, the distance between donor and acceptor must be within the range of resonance energy transfer phenomenon. However in the present case, the gold nanoclusters are synthesized in conjugation with the BSA molecules separately, and the BSA molecules are used for chemically exfoliation of the $\mathrm{MoS}_{2}$ nanosheets. This causes the enlargement of the distance between the gold nanoclusters and the $\mathrm{MoS}_{2}$ nanosheets, which limits the possibility of any kind of energy transfer, even though there is a overlap of the emission spectrum of gold nanoclusters and the absorption spectrum of the $\mathrm{MoS}_{2}$ nanosheets (see Figure 6). There is a complete overlap of A excitonic peak of $\mathrm{MoS}_{2}$ with the Fl emission peak of the gold nanoclusters, and partial overlap of the B excitonic peak. In the present case, the distance between the emitters and the acceptors is beyond the limit of the fluorescence resonance energy transfer, which is typically in the range of 1-10 nm. In case of gold nanoclusters and $\mathrm{MoS}_{2}$ nanosheets, the intermediate layer of the BSA molecules helps to increase the distance between emitters and acceptors beyond the permitted upper limit of the distance. This was further confirmed by using fluorescent dye molecules, Rhodamine 6G (R6G) as donor molecules in place of $\mathrm{Au}$ NCs. R6G molecules show Fl emission around $550 \mathrm{~nm}$, which has an overlap with the absorption spectrum of $\mathrm{MoS}_{2}$ (shown in Figure 6). Figure 7 shows the Fl lifetime decay curves of $\mathrm{R} 6 \mathrm{G}$ molecules in absence and presence of $\mathrm{MoS}_{2}$ nanosheets. Here the $\mathrm{MoS}_{2}$ nanosheets are same as the previous case, where BSA molecules are on surface of $\mathrm{MoS}_{2}$ and they act as stabilizing agent for the nanosheets. The Fl lifetime of R6G molecules is approximately 4 ns in absence of acceptors. In presence of $\mathrm{MoS}_{2}$ (BSA), there is no significant change in the $\mathrm{Fl}$ lifetime of R6G molecules, which is due to significant distance between R6G molecules and $\mathrm{MoS}_{2}$ nanosheets. This distance is suppose to be of the order of BSA molecule, which is 


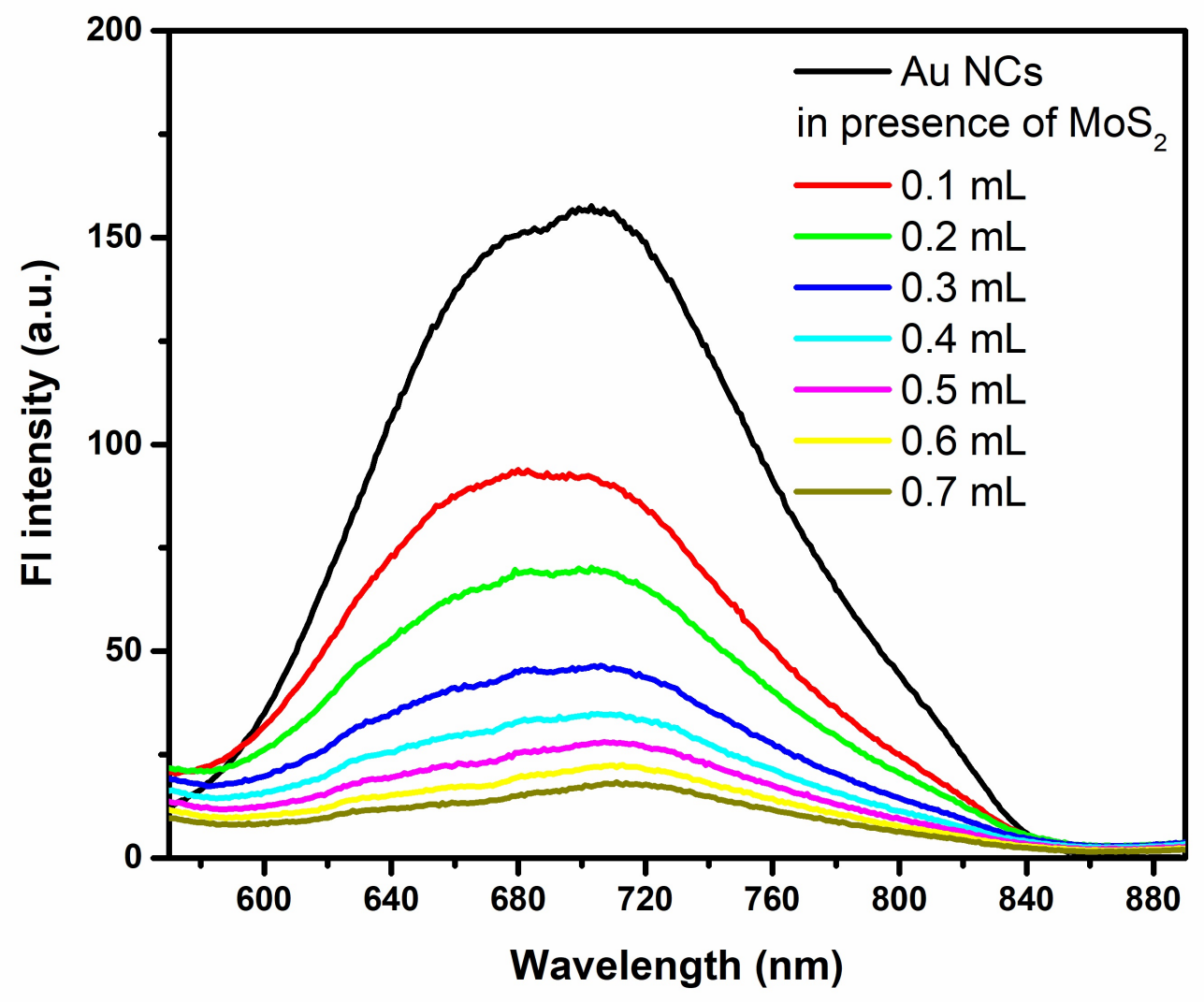

FIG. 4. Fluorescence spectra of gold nanoclusters in absence as well as in the presence of different quantity of $\mathrm{MoS}_{2}$ nanosheets.

acting as separator between $\mathrm{R} 6 \mathrm{G}$ and $\mathrm{MoS}_{2}$. In order to shorten the distance between R6G and $\mathrm{MoS}_{2}$, a new set of $\mathrm{MoS}_{2}$ nanosheets has been prepared using n-methyl-2-pyrrolidone (NMP) as dispersion medium. In NMP solution, $\mathrm{MoS}_{2}$ powder was dispersed and the mixture was sonicated for $20 \mathrm{~h}$. The supernatant was collected and used as acceptor for energy transfer study. The Fl lifetime behaviors of R6G molecules in presence and absence of $\mathrm{MoS}_{2}$ nanosheets are shown in Figure 8 In Figure 8 a significant change in the Fl lifetime of R6G molecule is observed. This indicates that there is energy transfer phenomenon taking place from $\mathrm{R} 6 \mathrm{G}$ molecules to the $\mathrm{MoS}_{2}$ nanosheets. In this case, the separation between $\mathrm{R} 6 \mathrm{G}$ and $\mathrm{MoS}_{2}$ nanosheets are within the limit of energy transfer distance $(1-10 \mathrm{~nm})$.

\section{SUMMARY}

In summary, the interaction of gold nanoclusters with the molybdenum disulfide was explored. It had been observed that the BSA, protein molecules, stabilized gold nanoclusters had strong florescence emission around $700 \mathrm{~nm}$ and its emission intensity can be altered in presence of $\mathrm{MoS}_{2}$ nanosheets. The florescence quenching was found to be static in the nature. It was observed when distance between donor and acceptor was short in case of R6G molecules and $\mathrm{MoS}_{2}$ nanosheets the energy transfer phenomenon took place. 


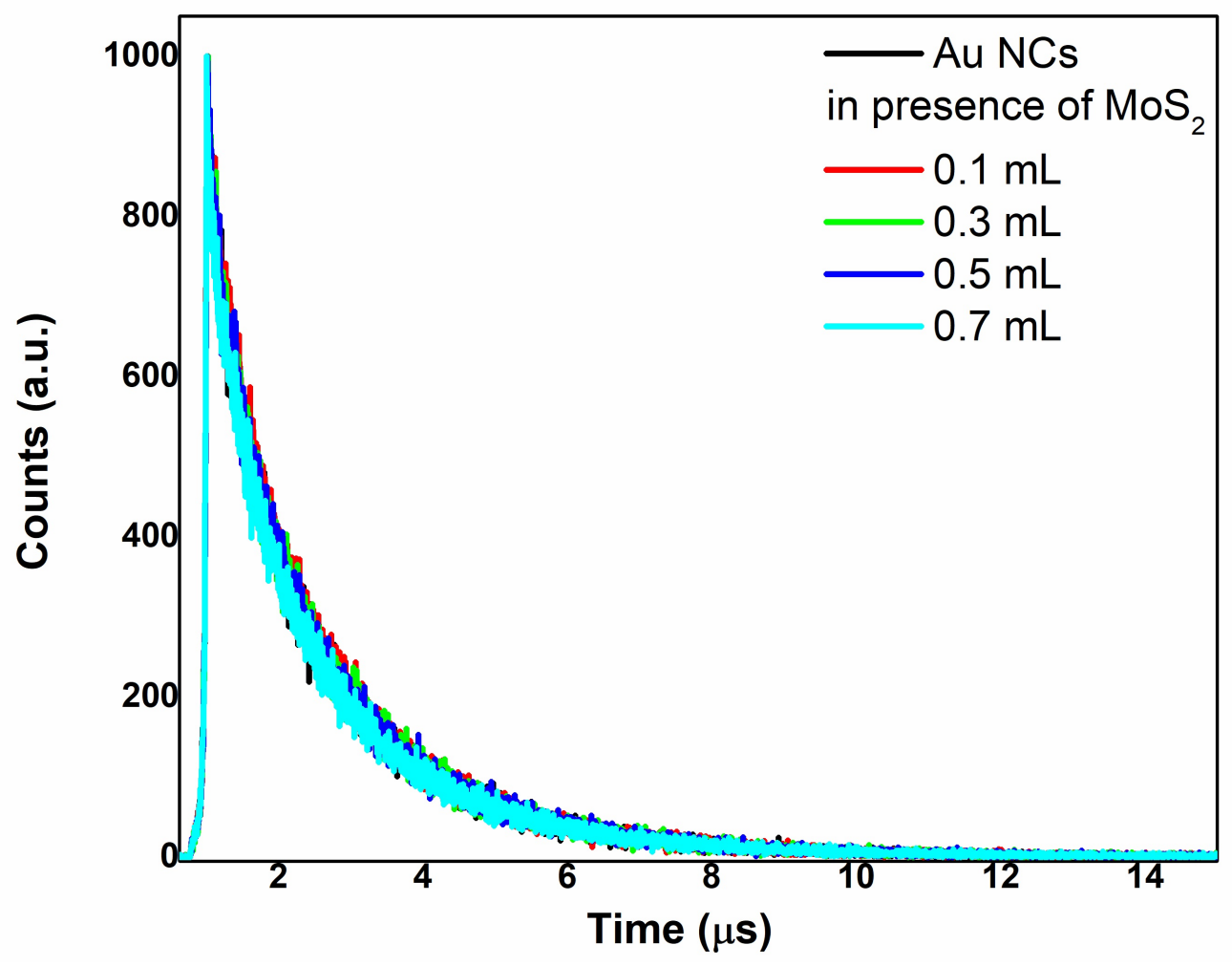

FIG. 5. Fluorescence lifetime decay curves of gold nanoclusters in absence and presence of different quantity of $\mathrm{MoS}_{2}$ nanosheets.

\section{ACKNOWLEDGEMENTS}

The authors are thankful to AIRF and SBT, JNU for characterization techniques.

${ }^{1}$ D. S. Yarramala, A. Baksi, T. Pradeep, and C. P. Rao, "Green synthesis of protein-protected fluorescent gold nanoclusters (auncs): Reducing the size of auncs by partially occupying the ca2 + site by la3+ in apo- $\alpha$-lactalbumin," ACS Sustainable Chemistry \& Engineering 5, 6064-6069 (2017).

${ }^{2}$ J. Lian, Q. Liu, Y. Jin, and B. Li, "Histone-dna interaction: an effective approach to improve the fluorescence intensity and stability of dna-templated cu nanoclusters," Chemical Communications 53, $12568-12571(2017)$

${ }^{3} \mathrm{X}$. Hu, Y. Zheng, J. Zhou, D. Fang, H. Jiang, and X. Wang, "Silver-assisted thiolate ligand exchange induced photoluminescent boost of gold nanoclusters for selective imaging of intracellular glutathione," Chemistry of Materials 30, 1947-1955 (2018).

${ }^{4}$ M. A. Abbas, P. V. Kamat, and J. H. Bang, "Thiolated gold nanoclusters for light energy conversion," ACS Energy Letters 3, 840-854 (2018).

${ }^{5}$ K. Pyo, V. D. Thanthirige, K. Kwak, P. Pandurangan, G. Ramakrishna, and D. Lee, "Ultrabright luminescence from gold nanoclusters: rigidifying the au (i)-thiolate shell," Journal of the American Chemical Society 137, 8244-8250 (2015).

${ }^{6}$ A. S. Patel and T. Mohanty, "Silver nanoclusters in bsa template: a selective sensor for hydrogen peroxide," Journal of materials science 49, 2136-2143 (2014).

${ }^{7}$ A. S. Patel, H. Sahoo, and T. Mohanty, "Understanding the interactions involved in the formation of fluorescent silver nanoclusters," Journal of Nanoscience and Nanotechnology 16, 8246-8251 (2016).

${ }^{8}$ J. Zheng, P. R. Nicovich, and R. M. Dickson, "Highly fluorescent noble-metal quantum dots," Annu. Rev. Phys. Chem. 58, 409-431 (2007).

${ }^{9}$ H. Dong, W. Gao, F. Yan, H. Ji, and H. Ju, "Fluorescence resonance energy transfer between quantum dots and graphene oxide for sensing biomolecules," Analytical chemistry 82, 5511-5517 (2010).

${ }^{10}$ A. R. Clapp, I. L. Medintz, J. M. Mauro, B. R. Fisher, M. G. Bawendi, and H. Mattoussi, "Fluorescence resonance energy transfer between quantum dot donors and dye-labeled protein acceptors," Journal of the American Chemical Society 126, 301-310 (2004). 


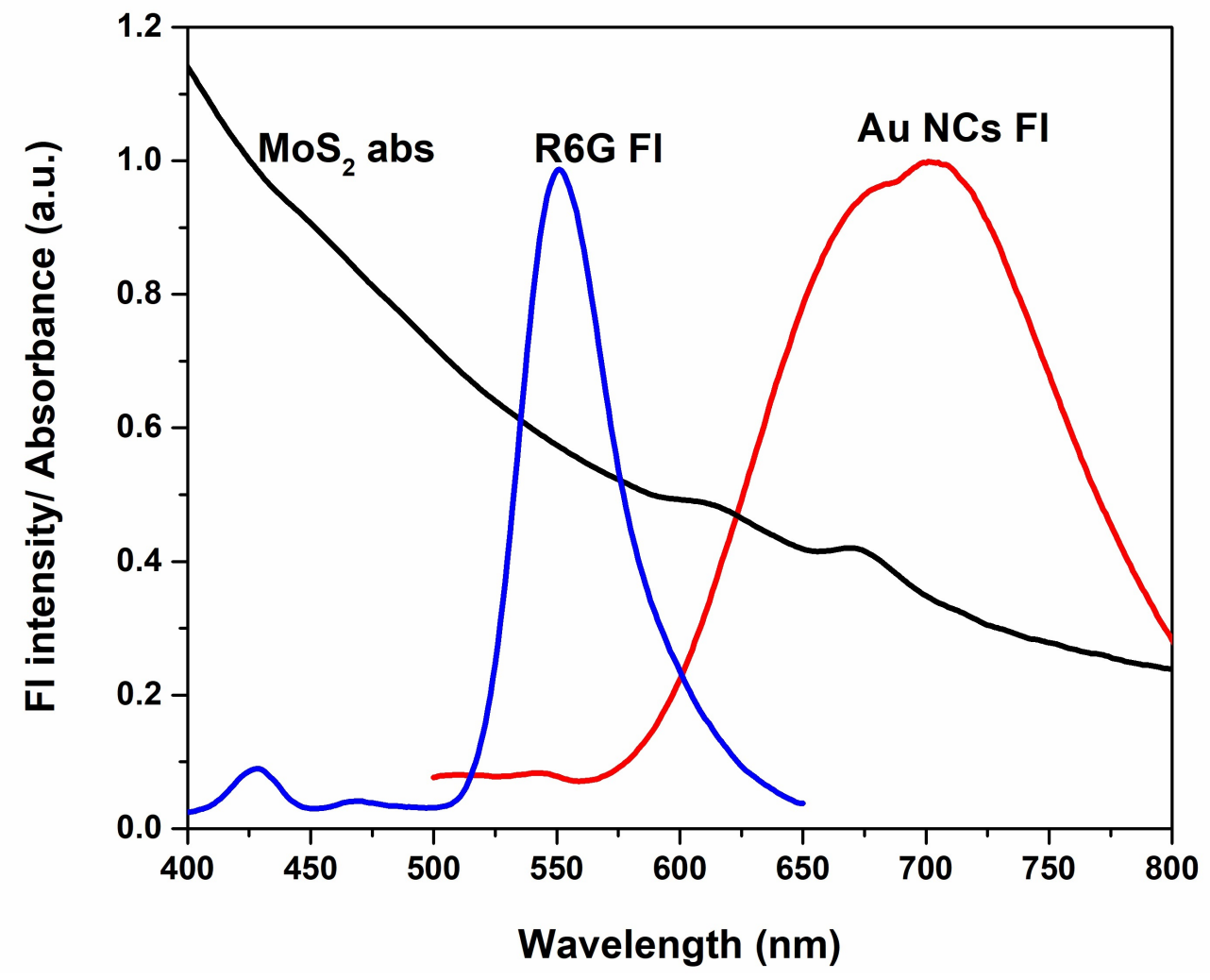

FIG. 6. Absorption spectrum of $\mathrm{MoS}_{2}$ nanosheets and emission spectra of gold nanoclusters and R6G molecules showing the overlapping of absorption and emission spectra.

${ }^{11} \mathrm{~S}$. Hohng and T. Ha, "Single-molecule quantum-dot fluorescence resonance energy transfer," ChemPhysChem 6, 956-960 (2005).

${ }^{12}$ D. Prasai, A. R. Klots, A. Newaz, J. S. Niezgoda, N. J. Orfield, C. A. Escobar, A. Wynn, A. Efimov, G. K. Jennings, S. J. Rosenthal, et al., "Electrical control of near-field energy transfer between quantum dots and two-dimensional semiconductors," Nano letters 15, 4374-4380 (2015).

${ }^{13}$ K. M. Goodfellow, C. Chakraborty, K. Sowers, P. Waduge, M. Wanunu, T. Krauss, K. Driscoll, and A. N. Vamivakas, "Distance-dependent energy transfer between cdse/cds quantum dots and a two-dimensional semiconductor," Applied Physics Letters 108, 021101 (2016).

${ }^{14} \mathrm{~F}$. Prins, A. J. Goodman, and W. A. Tisdale, "Reduced dielectric screening and enhanced energy transfer in single-and few-layer mos2," Nano letters 14, 6087-6091 (2014).

${ }^{15}$ M. Chhowalla, H. S. Shin, G. Eda, L.-J. Li, K. P. Loh, and H. Zhang, "The chemistry of two-dimensional layered transition metal dichalcogenide nanosheets," Nature chemistry 5, 263 (2013).

${ }^{16}$ Q. H. Wang, K. Kalantar-Zadeh, A. Kis, J. N. Coleman, and M. S. Strano, "Electronics and optoelectronics of two-dimensional transition metal dichalcogenides," Nature nanotechnology 7, 699 (2012).

${ }^{17}$ G. Guan, S. Zhang, S. Liu, Y. Cai, M. Low, C. P. Teng, I. Y. Phang, Y. Cheng, K. L. Duei, B. M. Srinivasan, et al., "Protein induces layer-by-layer exfoliation of transition metal dichalcogenides," Journal of the American Chemical Society 137, 6152-6155 (2015).

${ }^{18}$ F. Wu, Y. Xia, M. Sun, and A. Xie, "Two-dimensional (2d) few-layers ws2 nanosheets: An ideal nanomaterials with tunable electromagnetic absorption performance," Applied Physics Letters 113, 052906 (2018).

${ }^{19}$ S. Luo, S. Dong, C. Lu, C. Yu, Y. Ou, L. Luo, J. Sun, and J. Sun, "Rational and green synthesis of novel two-dimensional ws $2 / \operatorname{mos} 2$ heterojunction via direct exfoliation in ethanol-water targeting advanced visible-light-responsive photocatalytic performance," Journal of colloid and interface science 513, 389-399 (2018).

${ }^{20}$ J. Shakya, A. S. Patel, F. Singh, and T. Mohanty, "Composition dependent fermi level shifting of au decorated mos2 nanosheets," Applied Physics Letters 108, 013103 (2016).

${ }^{21}$ H. S. Lee, S.-W. Min, Y.-G. Chang, M. K. Park, T. Nam, H. Kim, J. H. Kim, S. Ryu, and S. Im, "Mos2 nanosheet phototransistors with thickness-modulated optical energy gap," Nano letters 12, 3695-3700 (2012). 


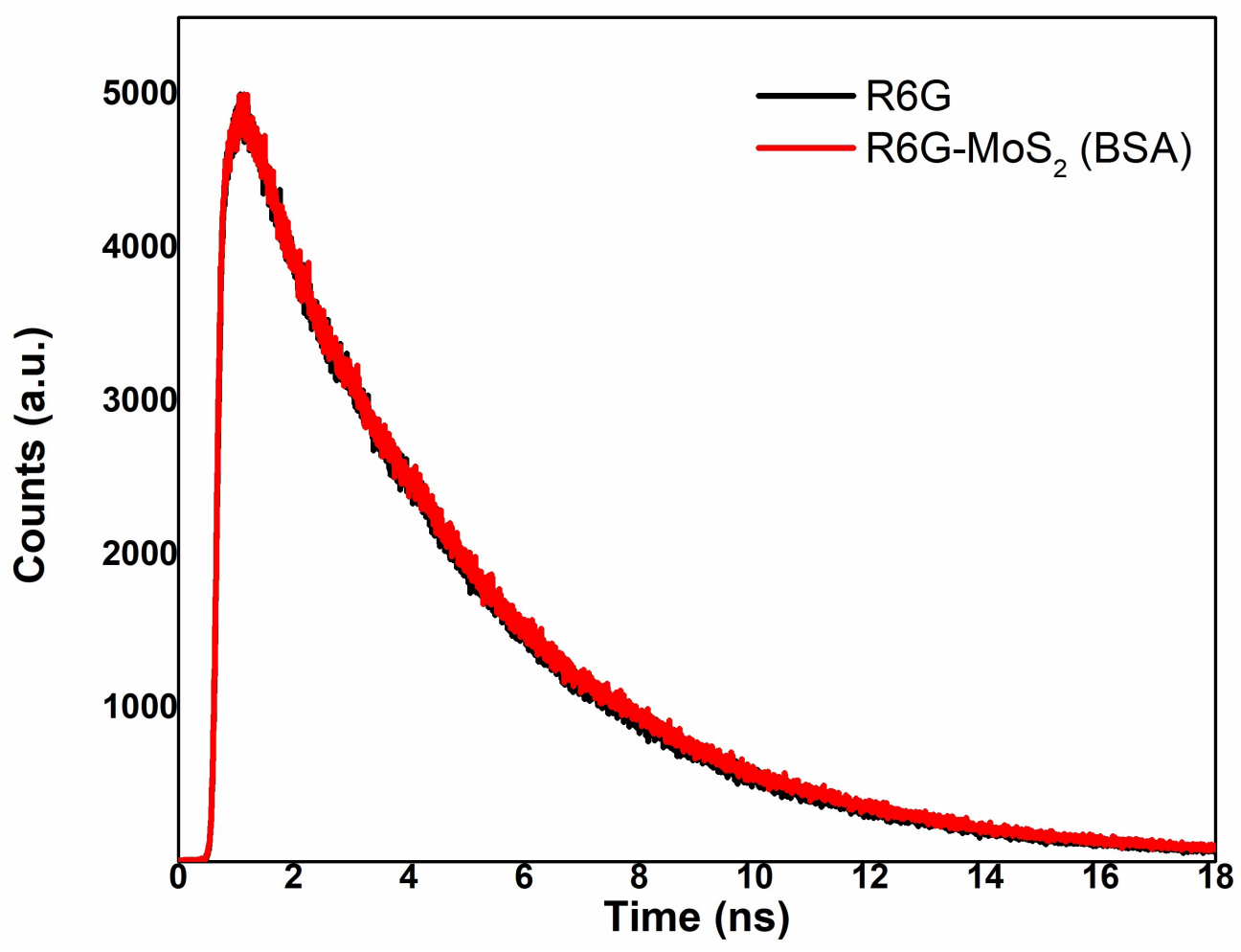

FIG. 7. Fluorescence lifetime decay curves of R6G molecules in absence and presence of $\mathrm{MoS}_{2}$ nanosheets, here $\mathrm{MoS}_{2}$ nanosheets are exfoliated using BSA molecules.

${ }^{22}$ A. Chakraborti, A. S. Patel, P. K. Kanaujia, P. Nath, G. V. Prakash, and D. Sanyal, "Resonance raman scattering and ab initio calculation of electron energy loss spectra of mos2 nanosheets," Physics Letters A 380, 4057-4061 (2016).

${ }^{23}$ K. Srinivasan, K. Subramanian, K. Murugan, G. Benelli, and K. Dinakaran, "Fluorescence quenching of mos 2 nanosheets/dna/silicon dot nanoassembly: effective and rapid detection of hg $2+$ ions in aqueous solution," Environmental Science and Pollution Research , 1-10 (2018).

${ }^{24}$ J. I. Paredes, J. M. Munuera, S. Villar-Rodil, L. Guardia, M. Ayan-Varela, A. Pagán, S. D. AznarCervantes, J. L. Cenis, A. Martínez-Alonso, and J. M. Tascon, "Impact of covalent functionalization on the aqueous processability, catalytic activity, and biocompatibility of chemically exfoliated mos2 nanosheets," ACS applied materials \& interfaces 8, 27974-27986 (2016).

${ }^{25}$ G.-H. Lee, Y.-J. Yu, X. Cui, N. Petrone, C.-H. Lee, M. S. Choi, D.-Y. Lee, C. Lee, W. J. Yoo, K. Watanabe, et al., "Flexible and transparent mos2 field-effect transistors on hexagonal boron nitride-graphene heterostructures," ACS nano 7, 7931-7936 (2013).

${ }^{26}$ A. S. Patel, H. Sahoo, and T. Mohanty, "Investigating the energy transfer from dye molecules to dna stabilized au nanoparticles," Journal of fluorescence 26, 1849-1855 (2016).

${ }^{27}$ P. P. H. Cheng, D. Silvester, G. Wang, G. Kalyuzhny, A. Douglas, and R. W. Murray, "Dynamic and static quenching of fluorescence by $1-4 \mathrm{~nm}$ diameter gold monolayer-protected clusters," The Journal of Physical Chemistry B 110, 4637-4644 (2006).

${ }^{28}$ A. S. Patel, H. Sahoo, and T. Mohanty, "Probing the förster resonance energy transfer between fluorescent copper nanoclusters and cobalt complex," Applied Physics Letters 105, 063112 (2014).

${ }^{29}$ A. S. Patel, P. Mishra, P. K. Kanaujia, S. S. Husain, G. V. Prakash, and A. Chakraborti, "Investigating resonance energy transfer from protein molecules to van der waals nanosheets," RSC Advances 7, 2625026255 (2017). 


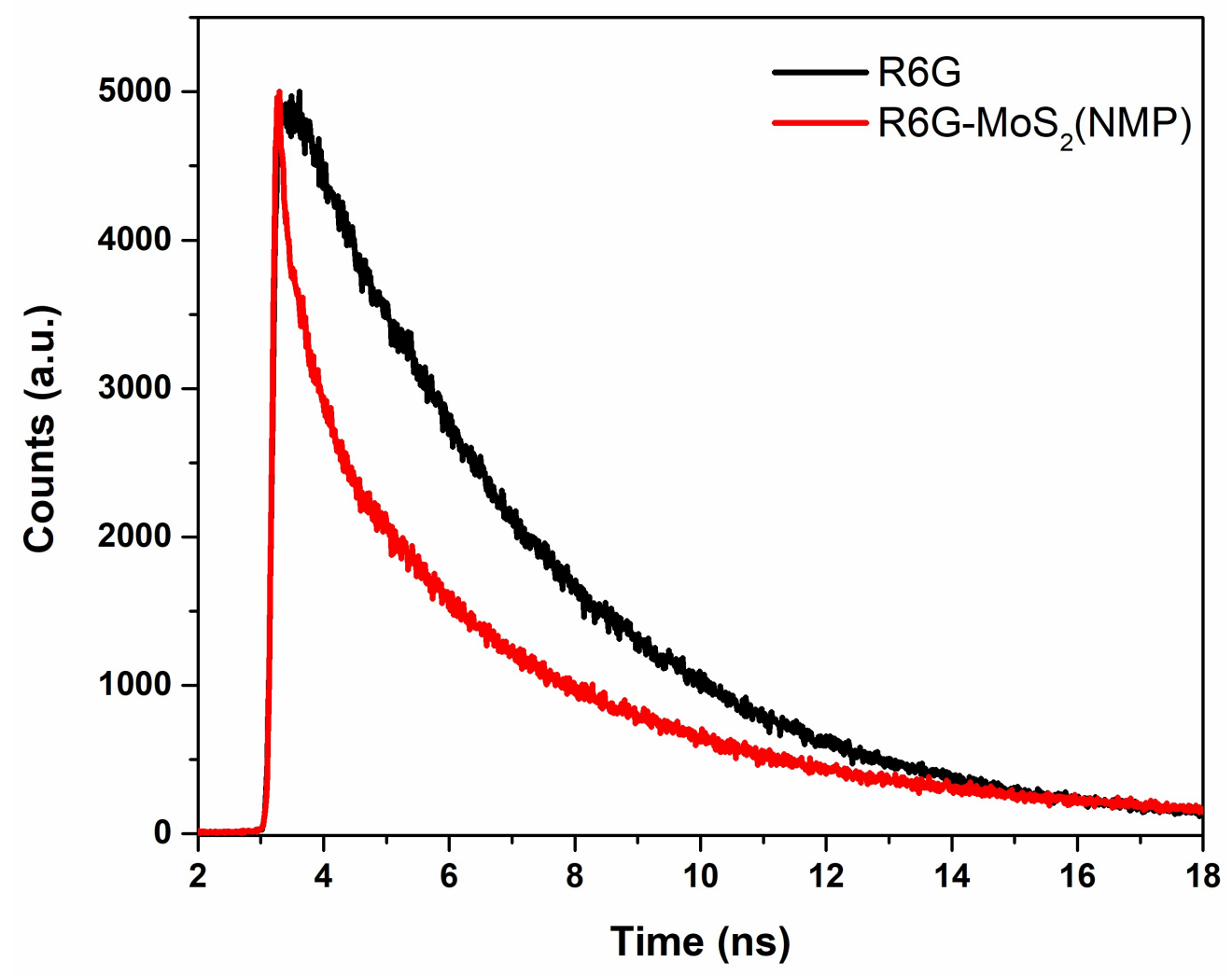

FIG. 8. Fluorescence lifetime decay curves of R6G molecules in absence and presence of $\mathrm{MoS}_{2}$ nanosheets, here $\mathrm{MoS}_{2}$ nanosheets are exfoliated in NMP solvent. 